This item was submitted to Loughborough's Research Repository by the author.

Items in Figshare are protected by copyright, with all rights reserved, unless otherwise indicated.

\title{
Accuracy of energy prediction methodologies
}

PLEASE CITE THE PUBLISHED VERSION

http://dx.doi.org/10.1109/WCPEC.2006.279946

PUBLISHER

(c) IEEE

VERSION

VoR (Version of Record)

LICENCE

CC BY-NC-ND 4.0

\section{REPOSITORY RECORD}

Williams, Sheryl R., Thomas R. Betts, Ralph Gottschalg, David Infield, H. de Moor, N. van der Borg, A.R. Burgess, et al.. 2019. "Accuracy of Energy Prediction Methodologies". figshare. https://hdl.handle.net/2134/8204. 
This item was submitted to Loughborough's Institutional Repository (https://dspace.lboro.ac.uk/) by the author and is made available under the following Creative Commons Licence conditions.

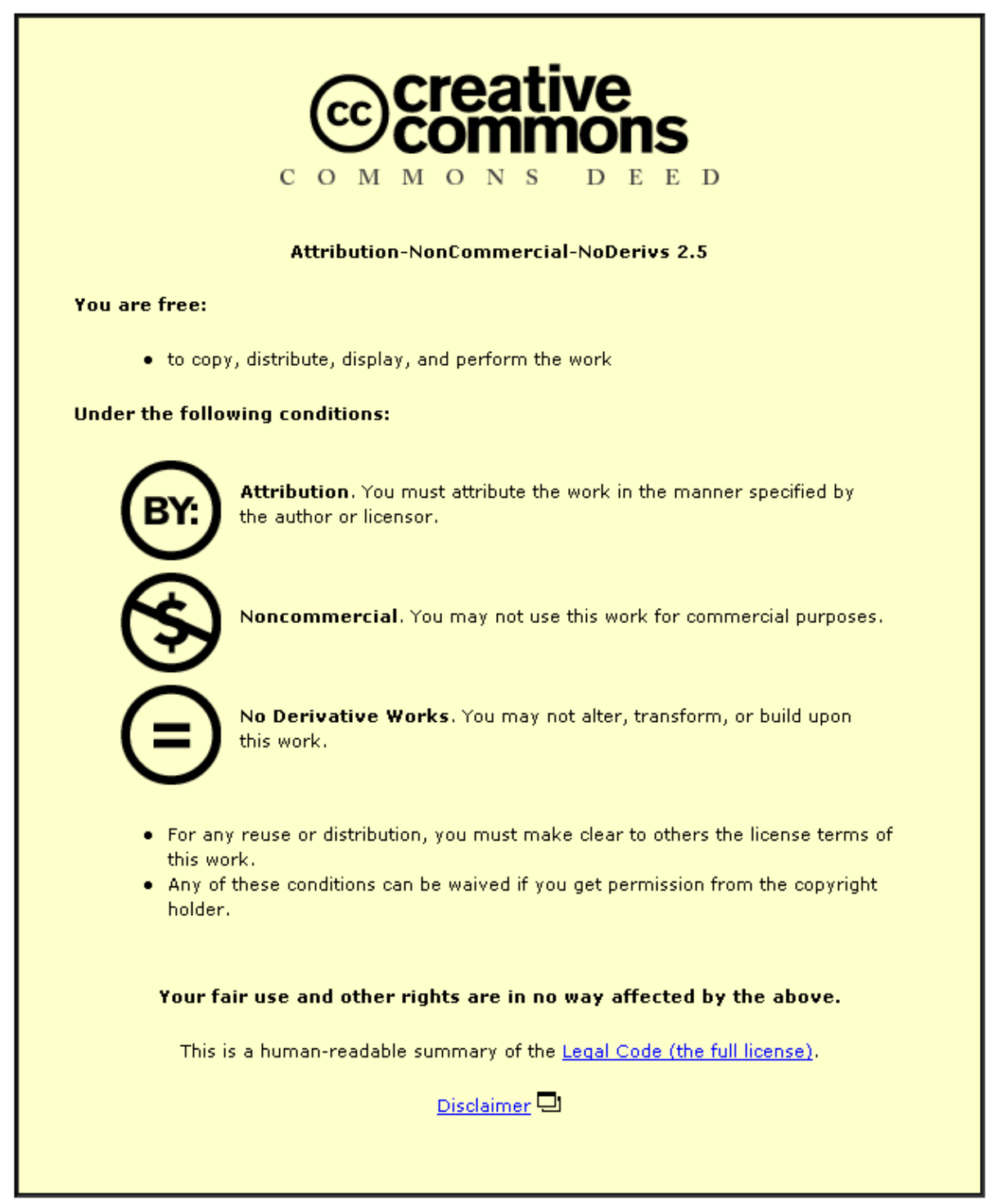

For the full text of this licence, please go to: http://creativecommons.org/licenses/by-nc-nd/2.5/ 


\title{
ACCURACY OF ENERGY PREDICTION METHODOLOGIES
}

\author{
S.R. Williams ${ }^{1 *}$, T.R.Betts ${ }^{1}$, R. Gottschalg ${ }^{1}$, D.G. Infield ${ }^{1}$, H. de Moor $^{2}$, N. van der Borg ${ }^{2}$, A.R. Burgers ${ }^{2}$; G. Friesen ${ }^{3}$ \\ D. Chianese ${ }^{3}$, A. Guerin de Montgareuil ${ }^{4} ;$ T. Zdanowicz ${ }^{5}$; D. Stellbogen ${ }^{6 ;}$ W. Herrmann ${ }^{7}$
}

${ }^{1}$ Centre for Renewable Energy Systems Technology, Loughborough University,UK; ${ }^{2} \mathrm{ECN}$, Petten, NL; ${ }^{3} \mathrm{SUPSI}$, Cannobio, $\mathrm{CH} ;{ }^{4} \mathrm{GENEC}$, Caderache, FR; $;$ Wroclaw University of Technology, Wroclaw, PL; ${ }^{6} \mathrm{ZSW}$, Stuttgart, D $;{ }^{7} \mathrm{TÜV}$ Rheinland, Cologne, D. *Corresponding author: S.R.Williams@lboro.ac.uk

\section{ABSTRACT}

In the current market, the specific annual energy yield $(\mathrm{kWh} / \mathrm{kWp})$ of a PV system is gaining in importance due to its direct link to the financial returns for possible investors who typically demand an accuracy of $5 \%$ in this prediction. This paper focuses on the energy prediction of photovoltaic modules themselves, as there have been significant advances achieved with module technologies which affect the device physics in a way that might force the revisiting of device modelling.

The paper reports the results of a round robin based evaluation of European modelling methodologies. The results indicate that the error in predicting energy yield for the same module at different locations was within $5 \%$ for most of the methodologies. However, this error increased significantly if the nominal nameplate rating is used in the characterization stage. For similar modules at the same location the uncertainties were much larger due to module-module variations.

\section{INTRODUCTION}

This paper reports the final results of the round robin tests of European modelling methodologies carried out during the Co-ordinated Action PVCatapult. In the first round robin[1],the accuracy and suitability of different modelling approaches were reviewed for year-year (predicted year) accuracy as well as the ability of predicting the energy yield using the same meteorological set as the one used for the extraction of the coefficients (base year) required for the different testing methodologies. The results provided an indication of how well the different methodologies handled temporal variations. For this the results were separated into three different photovoltaic technologies: CIS, amorphous double junction and crystalline. For the most part, the methodologies predicted energy yield within $5 \%$ for the base year and an increase of this to $10 \%$ for the predicted year. These results were fed back to the modelling groups involved in this round robin and it was investigated why certain methods seem to have higher deviations than others. The methods were upgraded and a second round robin, reported here, was set up. The longer term aim is to improve the modeling accuracy for the less simple devices (e.g. multi-junctions, operation-history dependent materials such as amorphous silicon). This requires a broader validation because at present most modelling methodologies are only tested for limited numbers of sites and technologies. It can be expected that different operating environments have significant potential to introduce prediction errors, as e.g. the latitude and weather conditions have a significant impact on the incident spectrum, resulting in a spectral mismatch which in turn directly impacts on the energy production and is normally not considered in common modelling approaches but crucial for multijunction devices, as shown below. This is not so much of an issue for crystalline silicon devices, as these are relatively narrow band gap material, however, other effects might be more important here. This round robin will be the basis fo9r further developments.

\section{Modelling Methods}

Modelling methodologies currently in use are based on a wide range of principles, e.g. some use measure-correlate-predict (e.g. Ransome and Wohlgemuth [2] and those based on physical device models [3]. In between there are models which parameterize laboratory measurements, matrix methods and realistic reporting conditions (RRC) approaches [4-7]. The methods studied under this project, are listed in Error! Reference source not found. and summarized below.

\begin{tabular}{|ll|}
\hline $\begin{array}{l}\text { Name of Modelling } \\
\text { Method }\end{array}$ & Developed By \\
\hline Matrix & LEEE (Cannobio, CH) \\
\hline MOTHER & CEA (Caderache, FR) \\
\hline $\begin{array}{l}\text { On-Line Yearly Yield } \\
\text { Simulator }\end{array}$ & ECN (Petten, NL) \\
\hline SSE & CREST(Loughborough, \\
& UK) \\
\hline
\end{tabular}

Table I: List of the performance models reviewed in this work along with the location of their research centers.

Matrix Method: This approach uses a power matrix or performance surface as a function of irradiance and ambient temperature. The energy yield is then calculated by multiplication of this matrix with a climatic condition occurrence matrix.

Meteorological, Optical and Thermal Histories for Energy Rating in Photovoltaics (MOTHERPV): This is still an experimental method in development by CEA. It allows the prediction of the performance ratio (PR) for sites with a good knowledge of the frequency distribution function of the incoming energy and the module back temperature as functions of irradiance. 
On-Line Yearly Yield Simulator: This method uses empirical laboratory translation for indoor to outdoor measured $\eta_{\text {mpp }}(G, T)$.

SSE (Site Specific Energy Rating): The SSE approach builds on the RRC method [3] and uses a measure-correlate-predict approach to model and predict the energy yield for PV modules.

The following sections outline the methodology, description of the dataset, results and discussion.

\section{METHODOLOGY AND DATASETS}

A two stage procedure was adopted to encompass the environmental and module-module variations. In stage one, the influence of module- module variation even within the same production batch was investigated to identify its influence on overall energy prediction. While data from the outdoor round robin, under the same Co-ordinated Action was used in stage two. Data of several short measurement campaigns of the same modules at different locations (ranging between 4-6 weeks) are used to exclude the module to module variation and purely focus on the effect of different environments.

\section{Stage 1: Module-Module Variations}

The data from the European Commission funded project 'Pythagoras' was used for stage 1 and the module-module variations of two thin film technologies were investigated for same site in the same year and for different site and different year. The block diagram in Figure 1 outlines the steps in this approach. The base module is used to provide the methodologies with the electrical measurements (I-V curves) and environmental data (irradiance in plane of array and back of module temperature) needed for characterization and the predicted module, as the name suggests, is the module for which the energy yield is predicted with only the environmental data.

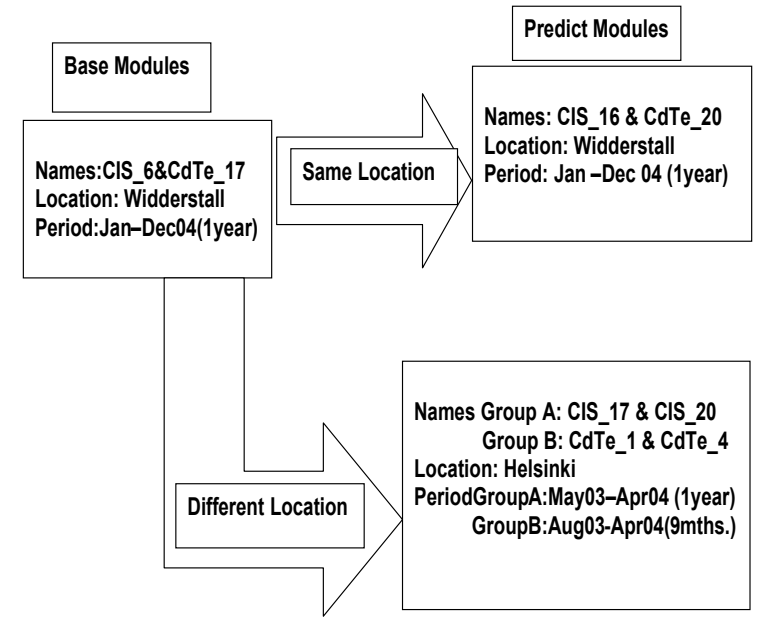

Figure 1: Block diagram with an overview of the dataset from the Pythagoras project

\section{Stage 2: Site-Site Variations}

The data used in stage 2 was the data generated as part of the outdoor measurement round robin under the same Co-ordinated Action, also reported at this conference [8]. The methodologies in this stage were assessed on the accuracy of the predicted energy yield for the same module at different environments. Several short measurement campaigns of the same module at different locations (with measurement times typically in the range of 2-6 weeks) are used to exclude the module to module variation and focus purely on the effect of different environments. This data was collected at several locations around Europe during the period April to December 2005.

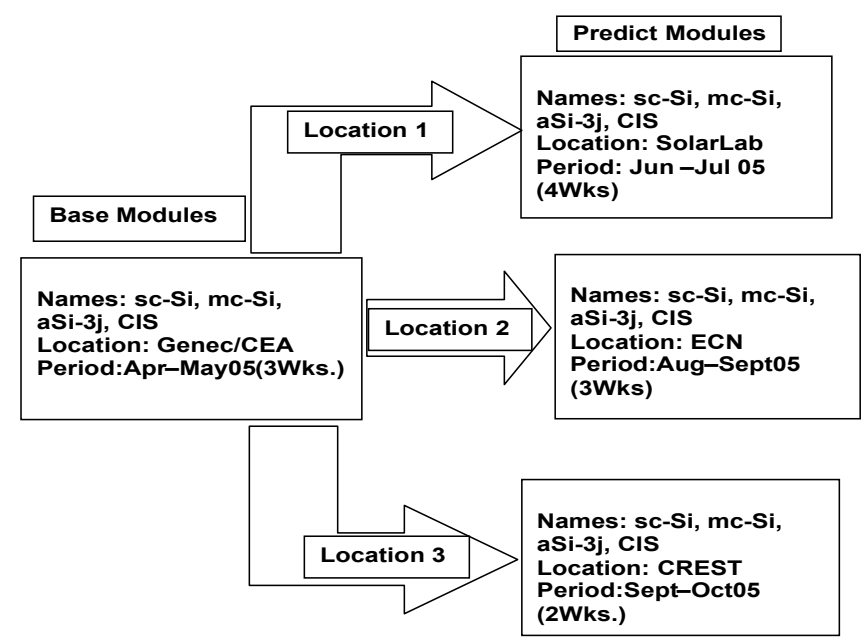

Figure 2: Block diagram with an overview of the dataset from PV-Catapult Measurement round robin.

Again, one site (CEA) was used as the source for the parameter extraction and characterization and the modules are referred to as base modules. The energy yield for these modules was then predicted by the methodologies at the different sites (SolarLab, ECN and CREST). Figure 2 outlines the procedure utilized in this stage.

A wide variety of module technology was used in this stage; they include single and multi crystalline silicon (sc-Si, ms-Si), triple junction amorphous silicon (aSi-3j) and copper indium diselenide (CIS).

\section{RESULTS}

The results generated from both stages of this round robin were systematically analyzed to identify the strengths and weaknesses of the studied modelling methodologies.

In stage 1, where the data from one module was translated to a similar module at the same site and period and to two similar modules at a different site and for a different period, the results were dominated by the module-module variations.

Figure 3 shows the accuracy of the energy yield prediction of the different methodologies for the CIS module, where CIS 6 is the base module, CIS 16 is a similar module at the same site and time, and CIS 17 $\& 20$ are similar modules at a different site and time. 
The average prediction error was in the order of $15 \%$, with virtually all methodologies under-predicting. This underestimation can be attributed largely to modulemodule variations, because the calculation of the energy yield of the base module resulted in an error of less than $3 \%$. The difference between site to site can be explained by occurrences of snow in the winter time, which certainly impacted on the prediction error. However, this error should be less than $5 \%$ because the base module was similarly affected. Thus, the large error is entirely due to the module-module variations where modules from the same batch can have variations in the order of several percent. One should also keep in mind, that the modules were not all installed at the same time and as 'Pythagoras' was also a project to demonstrate improvements, some of the modules will be - on purpose - better than the base module (which was one of the very first ones to be installed). In that sense, the under-prediction can actually be seen as a measure of how technology advanced (while the name-plate value remained the same) rather than be attributed to the modelling methodologies. This importance of the name plate value is demonstrated later again, when different approaches are taken to model the energy prediction of specific modules. MOTHER in this test accidentally used the electrical data from the different modules and demonstrates clearly that most of the variations are simply due to the lack of application of the electronic data.

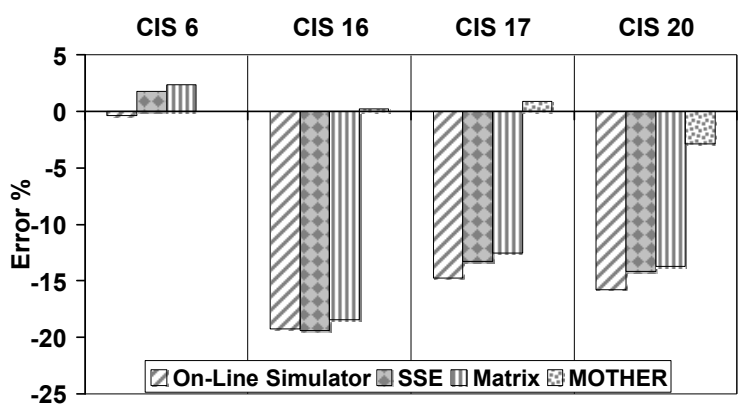

Figure 3: Accuracy of the energy yield prediction of $\mathrm{CIS}$ modules.

The energy yield of the CdTe as seen in Figure 4 is always over-predicted albeit less than $10 \%$ for most of the methods. All methods except MOTHER seem to have significant errors for these thin film devices. This would also be due to the different power rating (i.e. the rating actually delivered, rather than the nameplate value). If the real power can be derived, as in the case of the base module, the error drops for all methodologies to less than 4\%. Again, the good agreement of the MOTHER methodology can be seen as a confirmation of this.

SSE seems to be worst performer for these tests. The reasons were identified as being based on the name-plate efficiency rather than the actual one. The other methodologies are based on an initial determination of the efficiency of the base device (either explicitly or implicitly), which SSE did not have included in this step.
The difference between Online-Simulator and the other two methods (which are largely in line in their accuracy) cannot be explained. It appears, however, that the specific CdTe module type is very well suited for the online simulator.

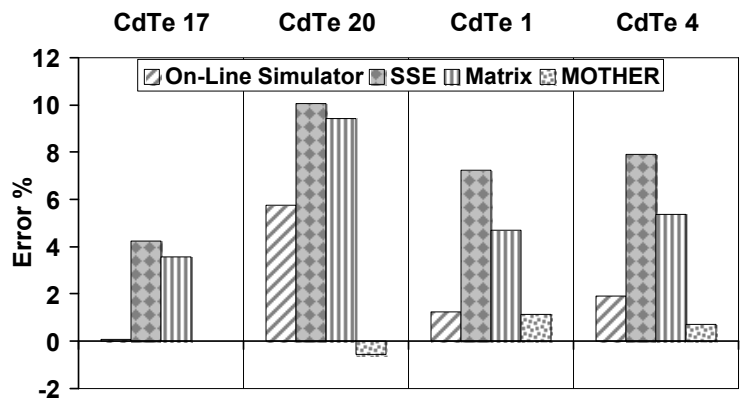

Figure 4: Accuracy of the energy yield prediction of CdTe modules.

In stage 2, site to site translations were performed. The first translation was the translation from the measurements of the CEA in France to calculate the energy yield at SolarLab in Poland, Figure 5 shows the error in the energy yield prediction for the different methodologies. In order to demonstrate the importance of the name plate efficiency, the SSE method was implemented in two ways: firstly, as indicated by an NP in brackets after the method in the legends below, using the nameplate efficiency of the modules under test, and secondly, using the efficiency of the module as determined from the CEA data (i.e. real power rating).

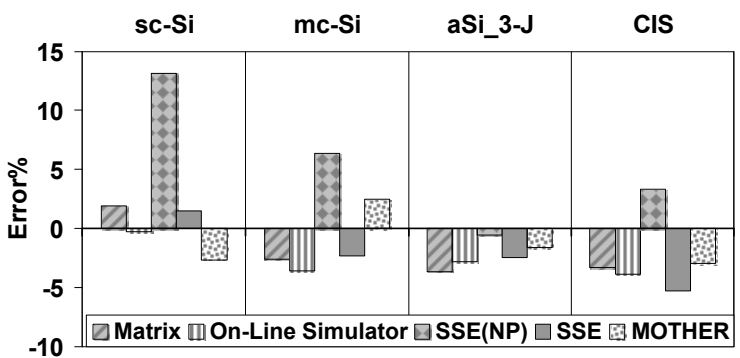

Figure 5: Accuracy of the energy yield prediction of the modules measured at SolarLab.

The results of this translation demonstrate clearly the importance of the nameplate efficiency. Using the name plate efficiency in all cases except for the a-Si triple junction and the CIGS module added a significant error to the accuracy of the energy prediction. All methods were able to predict the energy production of the modules to an accuracy of better than $4 \%$, with only SSE having one outlier in the case of CIS, which can be attributed to insufficient data filtering in the parameter generation phase for this particular module. Mostly the energy production is slightly under-predicted, with only the sc-Si module showing an overestimation. Looking at how the modules moved, the temperatures and intensities in Wroclaw will not have been so different to that at CEA, as we are later in the year but further north and thus average solar elevation is similar, which leaves 
spectrum and some late afternoon shading on some modules as the main reasons for the difference in accuracy for the various module types.

The translation to the Netherlands (ECN) as in Figure $\mathbf{6}$ is of even better quality than that to the SolarLab, but still there are no serious changes in the environment. No prediction for the Sc-Si is depicted for name-plate data, as this would have rendered the graph unreadable (there is more than 10 percent difference between name-plate and real power).

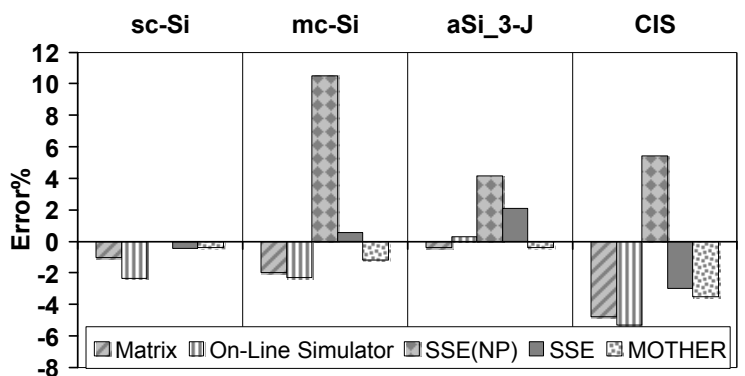

Figure 6: Accuracy of the energy yield prediction of the modules measured at ECN.

The online-simulator and the matrix method seem to have a significant increase in error for the CIGS, while still being close to the desired $5 \%$ mark. Some spectral differences will have occurred and while MOTHER and Matrix have them folded into other effects, SSE hasn't and thus the a-Si prediction is not as good as desired. The overall accuracy is not too astonishing as the module went further north with the year and are still very close to CEA in solar elevation and the operating temperatures are still very similar as the ambient temperatures are not too dissimilar from the CEA measurements. This similarity of the environment was broken when the modules went further north and the autumn arrived.

The energy prediction of the measurements at CREST showed a more significant error, as illustrated in Figure 7. SSE, which assumes independence of effects of each other seems to struggle with the wide band gap material, although this is expected as here a spectral correction needs to be applied. Overall, the accuracy of all models is still within the desired $5 \%$ margin but the different season shows.

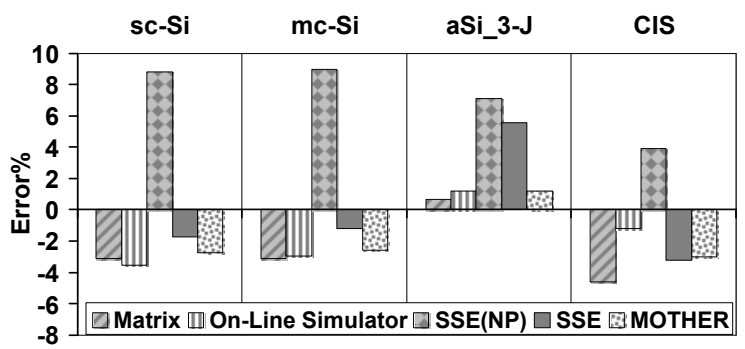

Figure 7: Accuracy of the energy yield prediction of the modules measured at CREST.

\section{CONCLUSIONS}

Stage 1 of the RR highlighted the difficulties with accurately predicting energy yield for similar modules due to module -module variations. There is a clear need for further research in this area

Stage 2 of the RR showed that all methods were able to predict the energy production of known modules in different environments very well. The comparison of name-plate and real rating carried out by CREST shows that the dominant factor in the energy prediction is the power rating. It should be emphasized, however, that the power rating of the different modules at the different sites in this stage varies typically by less then two percent, i.e. the differences between measurements and simulation cannot be attributed to measurement errors. The calculation of the energy production at different sites can then be carried out safely, as long as the input parameters are correct and it showed specifically that data-cleaning is absolutely crucial for accurate energy prediction. One should also highlight that very short measurement campaigns, giving only several weeks worth of data, were used to characterize the modules. This seems to be sufficient, but also the target datasets are very short and random errors would be reduced in longer term calculations.

Future round robins should carry out tests by starting with test meteorological data, in order to see how well the energy production can be predicted using this input.

\section{ACKNOWLEDGEMENTS}

This work was funded through the European Commission as part of the co-ordination action PV-Catapult (Grant No: 502775 (SES6)).

\section{REFERENCES}

1. Williams,S.R., et. al. Evaluating The State of The Art Of Photovoltaic Performance Modelling In Europe. 20th European Photovoltaic Solar Energy Conference. 2005. Barcelona.

2. Ransome, S.J. and J.H. Wohlgemuth. Understanding and Correcting kWh/kWp Measurements. in PV in Europe. 2002. Rome.

3. Gottschalg, R., et al. Translation and validation of Laboratory Measurements of Amorphous Silicon Devices to Real Operating Conditions. in 17th European Photovoltaic Solar Energy Conference. 2001. Munich.

4. Raicu, A., et al. Annual and Seasonal Energy Rating of mono-Si, a-Si and GaAs Test Cells for the USA by the RRC Method. in IEEE PVSC. 1991.

5. Kenny, R.P., et al. Energy Rating of PV Modules: Comparison of Methods AND Approach. in WCPEC. 2003. Osaka, Japan.

6. Anderson, et al Obtaining Module Energy Rating From Standard Laboratory Measurements. in 17th European Photovoltaic Solar Energy Conference. 2001. Munich, Germany.

7. Williams, S.R., et al. Modelling Real Annual PV Module Performance with Consideration to Spectral and Incidence Angle Effects. in 19th European Photovoltaic Solar Energy Conference. 2004. Paris, France.

8. Betts, T.R. et. al. Photovoltaic Performance Measurements in Europe: PVCatapult Round Robin Tests. $4^{\text {th }}$ World Conference on Photovoltaic Energy Conversion, 2006, Hawaii, this conference.

initiate 